\title{
PHOTOPIC SPECTRAL SENSITIVITIES OF THE RED AND THE YELLOW FIELD OF THE PIGEON RETINA
}

\author{
J. F. WORTEL, R. J. Wubbels and J. F. W. Nuboer \\ Laboratory of Comparative Physiology, State University of Utrecht, Jan van Galenstraat 40, 3572 LA \\ Utrecht, The Netherlands
}

(Receited 23 August 1983; in revised form 1 December 1983)

\begin{abstract}
The spectral sensitivities of the red field and the yellow field in the retina of the homing pigeon (Columba Livia) were determined on the basis of ERG responses. Between 450 and $550 \mathrm{~nm}$ the relative spectral sensitivity of the yellow field turned out to be higher than that of the red field. The results are in agreement with spectral sensitivity data, obtained by behavioural threshold procedures.
\end{abstract}

Spectral sensitivity ERG Retinal fields Oil-droplets

\section{INTRODUCTION}

The cones in the retina of probably all diurnal birds contain both coloured and colourless oil-droplets. The coloured oil-droplets act as cut-off filters, that reduce the short-wave sensitivity of the cones. In the pigeon the type of visual pigment in a cone can often be inferred from the colour of its oil-droplet (Bowmaker, 1977). Red and yellow droplets occur only in combination with a visual pigment that has a maximum absorption at $567 \mathrm{~nm}$. Orange droplets are only associated with a " $514 \mathrm{~nm}$ pigment", but greenyellow droplets may be associated with a 567 , a 514 or a $460 \mathrm{~nm}$ pigment. It is not known which oildroplet(s) are associated with the $413 \mathrm{~nm}$ pigment, that was demonstrated by Graf and Van Norren (1974), Van Norren (1975) and Govardovskii and Zueva (1977).

In the posterior dorsal part of the pigeon retina (the "red field") about $80 \%$ of the oil-droplets is red or orange as opposed to $25 \%$ in the remaining part of the retina (the "yellow field") (Waelchli, 1883). Therefore the red and the yellow field are characterized by different ratios of oil-droplet/cone-pigment combinations. In addition, the cut-off wavelengths of the oil-droplets in the red field are some $10 \mathrm{~nm}$ longer than those of similarly coloured droplets in the yellow field (Bowmaker, 1977).

On the basis of these anatomical differences the red and the yellow field can be expected to have different photopic spectral sensitivities. We have measured the photopic spectral sensitivity of the red and the yellow field electrophysiologically in two ways using ERG responses of anaesthetized pigeons: flicker photometry at a flicker rate of $30 \mathrm{~Hz}$ proved to be the most convenient and accurate way. To reveal contributions from cone systems with slow temporal characteristics a criterion response method (test-light frequency $1 \mathrm{~Hz}$ ) was used.

\section{MATERIALS AND METHODS}

\section{Subjects}

Seven adult homing pigeons (Columba Livia), male as well as female, were used in our experiments.

\section{Anaesthesia and immobilization}

Immediately before an experimental session the subject was anaesthetized by intramuscular injections of xylasine (Rompun, Bayer) $(6 \mathrm{mg} / \mathrm{kg}$ body weight) and ketamine hydrochloride (Ketaset, Bristol Laboratories) ( $60 \mathrm{mg} / \mathrm{kg}$ body weight) and placed in a bird holder. To enable stimulation of a ventral or dorsal part of the retina the bird was rotated $\alpha^{\circ}$ around a horizontal axis through the pupil parallel with the beak (Fig. 1). To stimulate nasal or temporal retinal fields the bird was rotated $\beta^{\circ}$ around a vertical axis through the pupil. Before application of corneal rings containing the electrodes to the eyes one drop of a local anaesthetic (Novesine, Union Pharmaceutique Belge) was administered to the cornea of each eye, In addition, one drop of a mydriatic (tubocurarine chloride) $(2.25 \mathrm{mg} / \mathrm{ml})$ in $0.025 \%$ benzalkonium chloride (Campell and Smith, 1962) was administered to the eye that was going to be stimulated.

\section{Stimulus system}

Flicker photometry (Fig. 1). A monochromatic test-light was alternated with a white reference-light and the intensity of the test-light was varied until the electrophysiological response amplitude was at a minimum. The reference-beam was delivered by a $50 \mathrm{~W}$ tungsten halogen source, which was somewhat underrun at a current of $4.1 \mathrm{~A}(10.7 \mathrm{~V})$. The test-light was obtained from a xenon-arc and a Bausch and Lomb grating monochromator (half-peak band width $19.2 \mathrm{~nm}$ ). To eliminate a short-wavelength overlapping order of light, a Corning cut-off filter (3-74) was used for wavelengths beyond $500 \mathrm{~nm}$. 


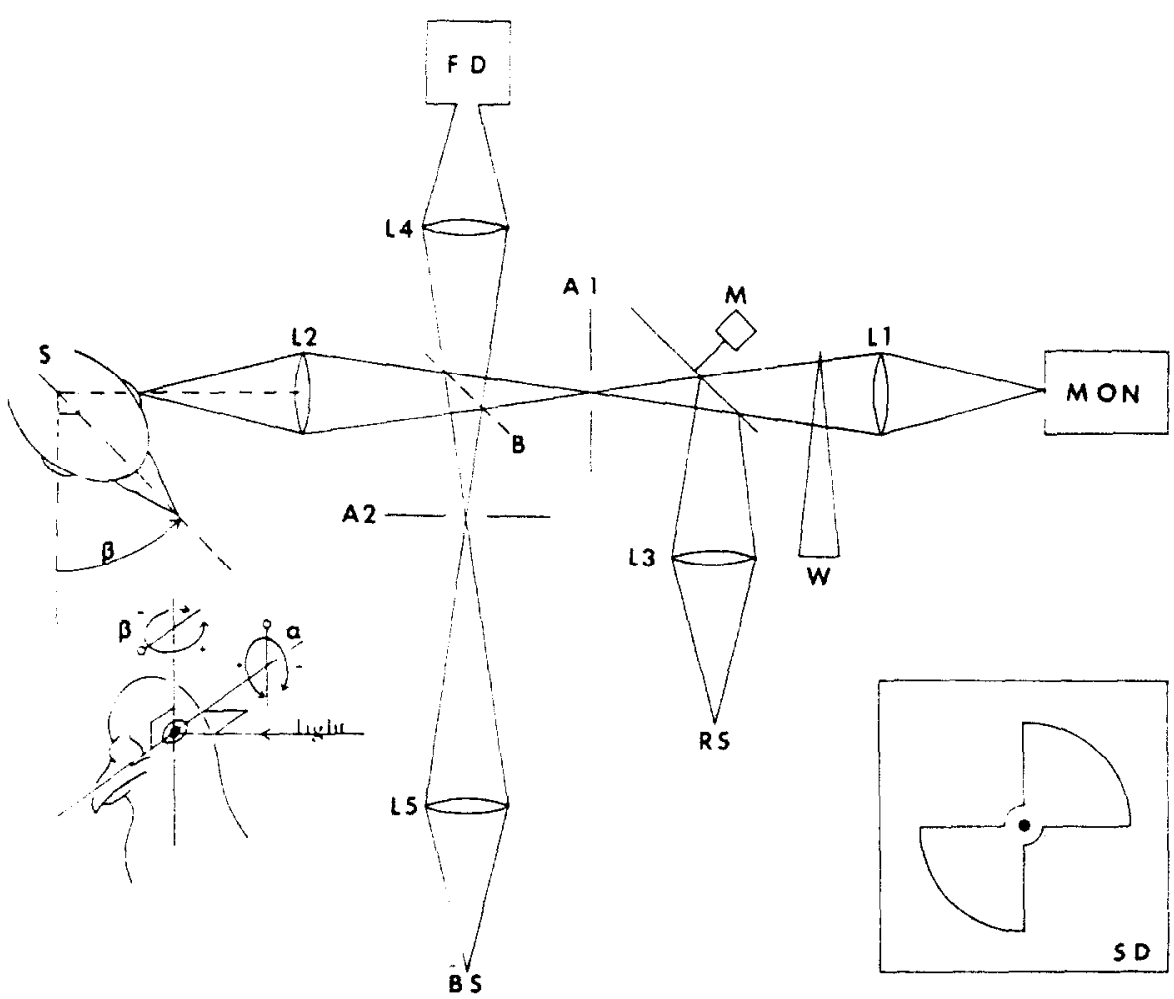

Fig. 1. Experimental set-up. A1. A2: circular apertures: B: beam-splitter; BS: background-source; FD: photo-diode; L1, L2, L3, L4, L5: lenses: M: motor driving the sector disc; MON: monochromator and xenon-source; RS: reference-source; S: subject; SD: sector disc; W: wedge.

Reference-light and test-light were chopped and combined by a rotating sector disc $(15 \mathrm{c} / \mathrm{sec})$ with a reflecting surface (Fig. 1, inset). The resulting stimulus was an alternation of 30 test- and 30 referenceflashes/sec. This high flicker frequency was chosen to eliminate rod responses. The stimulus beam illuminated a circular aperture (AI), which was imaged in the pupil of the subject, approximately $3.5 \mathrm{~mm}$ behind the corneal surface, where the nodal points of the eye are located (Marshall et al., 1973). The light beam of this Maxwellian view system subtended $12^{\circ}$. The image diameter was $2.1 \mathrm{~mm}$. Care was taken to keep the image totally within the dilated pupil and within the ring with the electrode.

Criterion response method. Whenever the criterion response method was used, the sector disc was removed and an electric shutter was placed in the stimulus beam near the circular aperture $\mathrm{Al}$. In this way a $200 \mathrm{~ms}$ test-flash was produced each second. To suppress any rod responses the retinal area stimulated by the test-light was also illuminated by a background-light provided by a $12 \mathrm{~V} / 4.3 \mathrm{~A}$ tungsten halogen source. The retinal illuminance resulting from the background light was $2.82 \log$ td for a human subject. If the spectral sensitivity of the pigeon were identical to that of man, this background would have provided a retinal illumination of $3.49 \mathrm{log}$ td in this bird, due to its shorter posterior nodal distance. In addition an annulus with a white, diffusely reflecting surface was positioned around lens L 2 .
The total diameter of this annulus subtended $40^{\circ}$. Its surface was illuminated by a $12 \mathrm{~V} / 50 \mathrm{~W}$ halogen source, filtered by a cinemoid daylight filter no. 45 , resulting in a luminance of $30 \mathrm{~cd} / \mathrm{m}^{2}$. In this way a $14^{\text {? }}$ wide annulus of retina surrounding the test stimulus was adapted to white light in order to reduce any contributions from responses to stray light. For a hypothetical pigeon with human spectral sensitivity the retinal illuminance of the annulus image was approximately $3.48 \log$ td.

\section{Recording and signal processing}

A thin chlorided silver wire, glued to the inner surface of a perspex ring, served as an electrode. The ring was placed on the cornea and was held in position by the eyelids, which were kept open by it. The ring did not prevent nictitating membrane blinks, which occurred occasionally even during anaesthesia. This was not very disturbing during the flicker photometry and appeared to be a convenient way of keeping the cornea moist. A second ring, placed on the unilluminated eye, served as a reference-electrode and the subject was grounded by a stainless steel needle, positioned subcutaneously in the neck.

Flicker photometry. The difference in the potential of the active- and the reference-electrode (ERG signal) was amplified by a condenser-coupled amplifier with a bandwidth of $1-300 \mathrm{~Hz}$. Averaging revealed that the ERG was roughly sinusoidal with a frequency of $30 \mathrm{~Hz}$. To improve the signal-to-noise ratio 


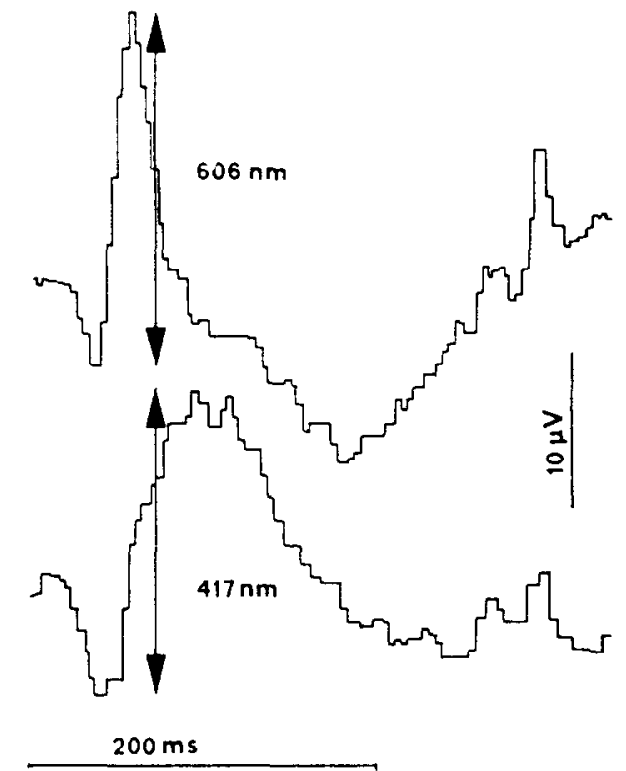

Fig. 2. Samples of averaged ERG responses as obtained using the criterion response method. Corneal positivity is recorded as an upward deflection. The stimulus wavelengths were $606 \mathrm{~nm}$ (upper record) and $417 \mathrm{~nm}$ (bottom record). The arrows pointing in two directions indicate the differences in potential, taken as the amplitudes of the $b$-components.

the ERG signal was cross-correlated with a stimulusderived square wave (HP $3721 \mathrm{~A}$ correlator), resulting in a nearly sinusoidal cross-correlogram (CCG) with a substantially lower noise level. The CCG produced by a test-light dominated stimulus differed by up to $180^{\circ}$ in phase with respect to a reference-light dominated CCG. When the ERG response to alternating test- and reference-flashes was at a minimum, the CCG amplitude was also at a minimum and its phase was intermediate between the phases of a test-light dominated and a reference-light dominated CCG. It proved convenient and accurate to use the intermediate phase of the CCG as a criterion for minimum ERG response (Nuboer et al., 1983).

Criterion response method. After amplification (bandwidth: $0.1-1000 \mathrm{~Hz}$ ) the ERG signal was filtered by means of an active filter (bandwidth: $0.5-80 \mathrm{~Hz}$; slope: $24 \mathrm{~dB}$ per octave) and averaged $(N=128)$. The parameter that we used was the difference in the potential of the (cornea-negative) peak of the $a$-wave and the (cornea-positive) peak of the $b$-wave (Fig. 2), which parameter can be considered as a good estimate of the $b$-component amplitude. Therefore the resulting spectral sensitivity is that of the $b$-component of the ERG.

\section{Calibration}

By means of a beam-splitter and the lens L4 (Fig. 1) part of the stimulus beam was focused on a reverse-biased photo-diode (SFD-160, EG\&G). After amplification the output of this device was used to calculate the radiant flux $F(\dot{\lambda})$ of a test-light with wavelength $\lambda$ (quanta/sec). The spectral sensitivity at wavelength $i$ was defined by: $S(\lambda)=1 F(j)$. The photo-diode was calibrated by means of a radiant flux meter system (HP 8330A/8334A). During this procedure the surface of the detector (a thermopile) was placed in the focus of the Maxwellian view system.

The luminance of the reference- and backgroundlight was measured by means of a luxmeter (Panlux, Gossen) according to the method described by $\mathrm{Ny}$ gaard and Frumkes (1982).

\section{Procedure}

Flicker photometry. During each experimental session four series of measurements were completed, consisting of 20 test-wavelengths each. The testwavelengths were used in the following order: $351-392-444-513-606, \quad 360-404-460-533-635 \mathrm{~nm}$ etc. (the corresponding test-wavenumbers were spaced $75 \mathrm{~mm}^{-1}$ apart). The red field was stimulated during the first two series $\left(z=25^{3} ; \beta=35^{3}\right)$, the yellow field during the last two series $\left(x=-15^{5}\right.$; $\beta=0^{\circ}$ ).

Before and after each series of measurements the ERG signal of the reference-light alone (referenceERG) was averaged. During all sessions the amplitude of the reference-ERG was hetween 1.3 and $5 \mu \mathrm{V}$ p-p. At equal stimulus levels the yellow field produced higher ERG amplitudes than the red field. Apparently the ERG flicker sensitivity of the yellow field was higher than that of the red field. Therefore before the yellow field was stimulated the intensity of the reference-light was attenuated as much as necessary to obtain a reference-ERG approximately equal to that during the red field measurements. For a hypothetical pigeon with human spectral sensitivity the retinal illuminance resulting from the reference-light was approximately $4.21 \mathrm{log}$ td during stimulation of the red field and $3.33 \mathrm{log}$ td during stimulation of the yellow field. The reference-intensity was not changed between two series of measurements of one retinal field.

Criterion response method. Because of the timeconsuming character of this method only one series of measurements, comprising 12 wavelengths, could be done during each experimental session. Either the red field $\left(x=25^{\circ} ; \beta=35^{\prime}\right)$ or the yellow field $\left(x=-15^{\prime} ; \beta=0^{\prime}\right)$ was stimulated. At every testwavelength the radiant flux required to obtain a criterion $b$-component amplitude was determined. We did this by interpolating two test-intensities that yielded $b$-component amplitudes near the criterion, assuming a linear relationship between the logarithm of the test-intensity and the amplitude of the $b$ component. Our own observations and those of Ikeda (1965) confirmed that this is a reasonable assumption, especially when only a short intensity range is considered. One of the 12 wavelengths served as a reference. The criterion was set by the $b$-component amplitude, evoked by a particular 
reference-intensity. This criterion amplitude was approximately $10 \mu \mathrm{V}$. Before and after every sensitivity determination (comprising two amplitude measurements at the same test-wavelength) the reference-amplitude was checked and the results were corrected for any change of the criterion.

\section{Ophthalmoscopic inspection of the retina}

By means of a hand-ophthalmoscope the temperodorsal part (red field) of the pigeon retina is seen to be more reddish-white than the remaining part of the retina, which has a greenish-white appearance. Using this colour difference we were able to select the angles $\alpha$ and $\beta$ that ensured stimulation of either the red or the yellow field. In addition, before and/or after measurements on a particular field, we verified the colour of that field as an additional control. For this purpose the lens L2 was removed and a semitransparent mirror was added to the set-up, making an angle of $45^{\circ}$ with the stimulus beam. The fundus was illuminated briefly by bright white light from the reference- or background-source, while the experimenter was observing the retina via the semitransparent mirror. The observations always confirmed that the field in question was stimulated. After every ophthalmoscopic inspection the semitransparent mirror was removed, the lens L.2 was put back in place and the bird was adapted for about $15 \mathrm{~min}$ to the reference-light level to be used in the next series of measurements.

Before and after several experimental sessions this simple ophthalmoscope was also used to determine the position of the tempero-dorsal end of the pecten $\left(\alpha=-29.4^{\circ} \pm 0.8^{\circ} ; \beta=23.4^{\circ} \pm 1.7^{\circ}\right)$. This was done to investigate whether significant eye-movements had

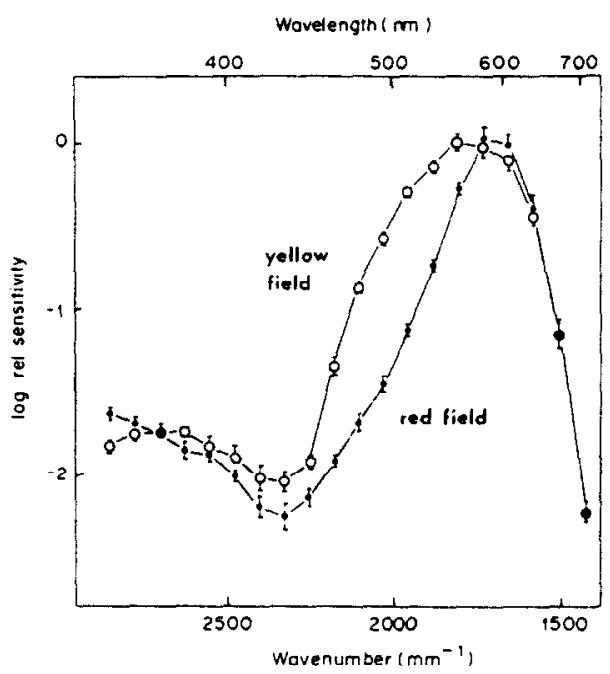

Fig. 3. Relative spectral sensitivity of the red field (solid circles) and the yellow field (open circles) of the pigeon retina, obtained by flicker photometry. Means of 10 observations and standard errors of the means (bars). The curves have been shifted along the ordinate to obtain the best mutual fit beyond $650 \mathrm{~nm}$.

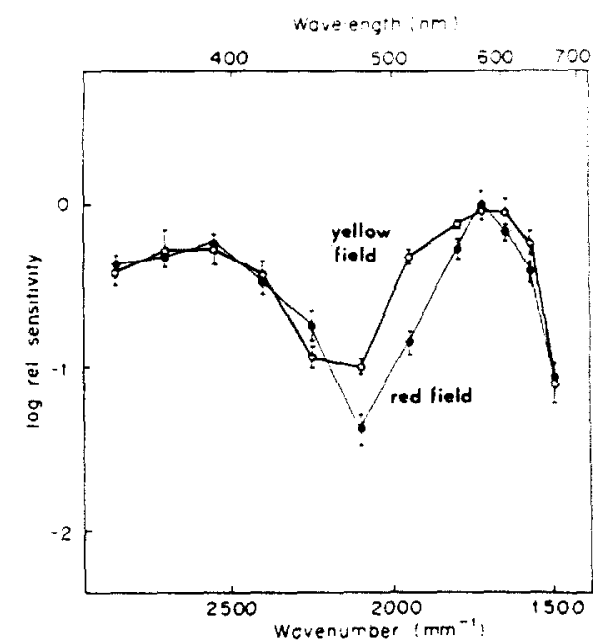

Fig. 4. Relative spectral sensitivity of the red field (solid circles) and the yellow field (open circles) of the pigeon retina, obtained by the criterion response method. Means of 5 observations and standard errors of the means (bars). The curves have been shifted along the ordinate to obtain the best mutual fit below $420 \mathrm{~nm}$ and beyond $650 \mathrm{~nm}$.

taken place during a session. Only minor differences were found in the angles $\alpha$ and $\beta$, as measured before and after a session.

\section{RESULTS}

Figure 3 shows the results of five experimental sessions with three pigeons, obtained by flicker photometry. To facilitate comparison the curves were shifted along the ordinate until they showed the best mutual fit beyond $650 \mathrm{~nm}$. In that spectral range the relative spectral sensitivities of the two retinal fields are identical as expected, because the incoming light is transmitted almost completely by all the oildroplets and it can be absorbed mainly by one visual pigment (absorption maximum at $567 \mathrm{~nm}$ ). However, in the spectral region between 450 and $550 \mathrm{~nm}$ the yellow field is up to 0.8 logarithmic units more sensitive than the red field relative to wavelengths beyond $650 \mathrm{~nm}$.

In Fig. 4 the results of the criterion response method are shown. The curves have been shifted vertically to obtain the best mutual fit beyond $650 \mathrm{~nm}$ as well as below $420 \mathrm{~nm}$, where the spectral sensitivity of both retinal fields is probably based principally on one visual pigment (absorption maximum at $413 \mathrm{~nm}$ ), associated with a colourless oil-droplet (see discussion). A comparison of the standard deviations of the means in Figs 3 and 4 shows that the results of the criterion response method show somewhat more statistical variation than those of ficker photometry. Nevertheless the difference in sensitivity in the spec. tral region between 450 and $550 \mathrm{~nm}$ is clearly confirmed using the criterion response method. A direct comparison of the absolute sensitivities of the two retinal fields by means of the criterion response 


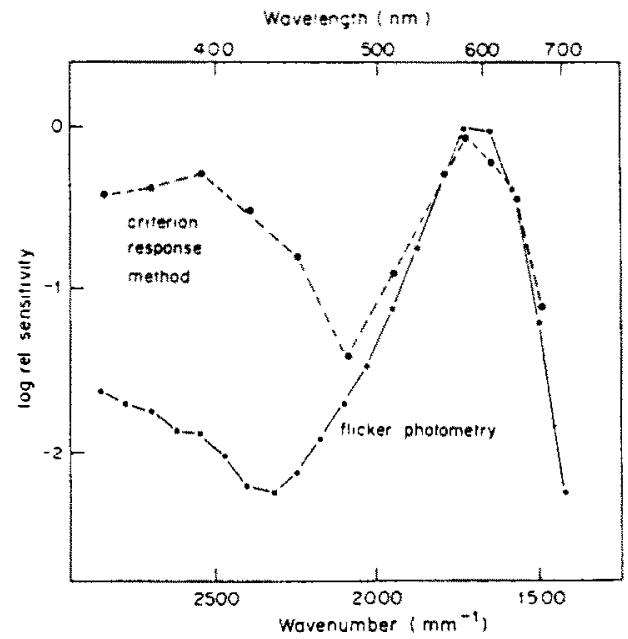

Fig. 5. Spectral sensitivity of the red field obtained by flicker photometry (full line) compared with that obtained by the criterion response method (solid circles and broken line). The curves were shifted along the ordinate to obtain the best mutal fit beyond $550 \mathrm{~nm}$.

method, in which the retinal field was varied but not the wavelength, revealed that the red field is approximately 0.26 logarithmic units more sensitive than the yellow field.

In Figs 5 and 6 the results of the criterion response method and flicker photometry are compared. The relative sensitivity beyond $476 \mathrm{~nm}$ does not differ significantly. However, the sensitivity to blue and violet light, as determined by the criterion response method, is much higher than that obtained by flicker photometry.

The waveform of the photopic pigeon ERG is somewhat dependent on the test-wavelength: when the stimulus wavelength is short (below about

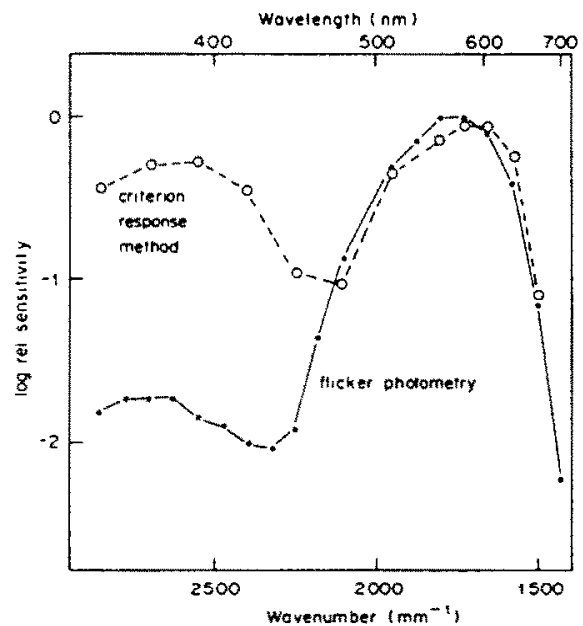

Fig. 6. Spectral sensitivity of the yellow field obtained by flicker photometry (full line) compared with that obtained by the criterion response method (open circles and broken line). The curves were shifted along the ordinate to obtain the best mutual fit beyond $550 \mathrm{~nm}$.
$450 \mathrm{~nm}$ ), the ERG components show long rise and fall times in comparison with a longer stimulus wavelength (Fig. 2). In addition, these components have longer latencies (Fig. 2; Graf and Van Norren, 1974). It seems that the violet sensitive cone-system has slower temporal characteristics than the remaining cone-systems. The same phenomenon has been reported for the turtle by Geri (1981). "Slow" receptor systems cannot follow high stimulus frequencies. Thus the violet sensitive cone-system will only have made a small contribution to the ERG responses obtained by flicker photometry, during which we stimulated the retina with as many as 60 light flashes/sec. This probably explains the large difference between the results of the criterion response method and flicker photometry in the short wavelength range of the spectrum.

\section{DISCUSSION}

Using a behavioural threshold procedure Martin and Muntz (1979) have demonstrated that the spectral sensitivity of the pigeon for a stimulus positioned above the horizontal of the lateral visual field is different from the spectral sensitivity for a stimulus positioned close to the line of sight along the bill. The authors assumed to have stimulated the yellow and the red field in this way.

However, Martinoya et al. (1981) demonstrated that a punctual light coming from a point in the sagittal plane $25^{\circ}$ below the direction of the beak is imaged near the centre of the red field. In view of this finding it must be doubted whether stimuli positioned close to the line of sight along the bill have their retinal images entirely within the red field, especially when it is realized that pigeons can make eyemovements of up to $15 \mathrm{deg}$ (Bloch et al., 1981). A comparison of our results with those of Martin and Muntz (Fig. 7) shows that their "red field" function is broader than ours, suggesting that Martin and Muntz did not stimulate the red field exclusively.

In Fig. 8 our results with the criterion method are compared with photopic spectral sensitivity functions obtained by Romeskie and Yager (1976), Blough (1957) and Graf and Van Norren (1974). Blough as well as Romeskie and Yager used a behavioural threshold procedure, but Graf and Van Norren used a criterion response method based on ERG responses to a flickering light $(25 \mathrm{~Hz})$. None of these authors did restrict the stimulus to either the red or the yellow field of the retina. The function of Romeskie and Yager shows a narrower peak occurring at a longer wavelength than do the curves of Blough and Graf and Van Norren. On the basis of this difference Romeskie and Yager have suggested that their function represents the red field sensitivity and that the other functions represent the yellow field sensitivity of the pigeon. This suggestion is confirmed by our results since the curve of Romeskie and Yager fits our red field function, while the curves of Graf and Van 


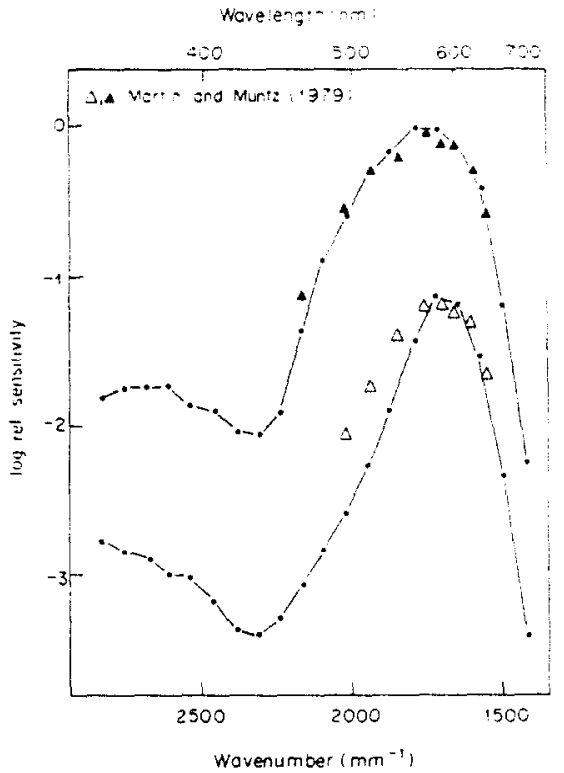

Fig. 7. Results of flicker photometry (lines) compared with spectral sensitivity functions obtained by Martin and Muntz (1979) supposed to correspond with the red and yellow field of the retina. The curves have been shifted along the ordinate to obtain the best mutual fit beyond $580 \mathrm{~nm}$ (red

field) or over the entire spectral range (yellow field).

Norren and Blough agree reasonably well with our yellow field function. However, especially Blough's results indicate a lower sensitivity to blue and violet light than we found. This discrepancy might be explained by the kind of light adaptation used by Blough, since he adapted his subjects immediately before the experiments to a "daylight" fluorescent light of high luminance $(914 \mathrm{~mL})$. The spectrum of these light sources is known to contain two important emission lines at about 405 and $435 \mathrm{~nm}$. Consequently Blough's pigeons must have been adapted to violet light during the performance of their task, resulting in a somewhat suppressed sensitivity to violet and blue light.

Thus our ERG results are in agreement not only with earlier electro-physiological data concerning the spectral sensitivity of the pigeon, but also with psychophysical threshold determinations. They leave little doubt that between 450 and $550 \mathrm{~nm}$ the photopic relative spectral sensitivities of the red and yellow field are really different. It could be argued that the difference is due to a contribution to the ERG by rods in the yellow field, possibly in response to stray light. This possibility seems unlikely, however, due to the high stimulation frequency during flicker photometry and the large-field adaptation in the criterion response method.

In view of the strong absorption of violet and blue light by the oil-droplets the criterion response method reveals a surprisingly high sensitivity to violet light. Flicker photometry selectively reduces the short-wave sensitivity, but not the long-wave sensitivity (Fig. 3). Apparently the long-wavelength sensitive cone sys- tems do not contribute considerably to the "violet" sensitivity. Therefore, violet sensitivity must be mediated by the $413 \mathrm{~nm}$ or the $460 \mathrm{~nm}$ pigment. Evidence, obtained by Bowmaker (1977) indicates that cones with the $460 \mathrm{~nm}$ pigment also contain a coloured oil-droplet, that strongly absorbs below $476 \mathrm{~nm}$. Therefore it seems likely that the "violel" sensitivity of the pigeon is mediated mainly by the $413 \mathrm{~nm}$ pigment. If cones with this visual pigment contain any oil-droplets at all, these will probably be colourless, since they are the only oil-droplets that seem to be transparent to violet light (Goldsmith and Collins, 1982). Thus the absorption maximum of this oil-droplet/cone-pigment combination should also be located at a wavelength of $413 \mathrm{~nm}$. It is remarkable therefore that the experimental sensitivity maximum lies at a shorter wavelength (Fig. 4). This might be an indication for antagonistic interactions at the level of the inner nuclear layer, resulting in inhibition of the violet sensitive cone-system by longer wavelength sensitive cone-systems within the spectral range in which both systems are sensitive. In the thalamus of the pigeon blue/yellow units have been demonstrated by Yazulla and Granda (1973), indicating that antagonistic interactions occur at least at a more central level

Part of the short-wave sensitivity may be due to fluorescence. Near-ultraviolet light can cause fluorescence of the yellow oil-droplets (Marc, cited by Donovan, 1978) and the emitted light might have stimulated long-wave cone-pigments. However, this

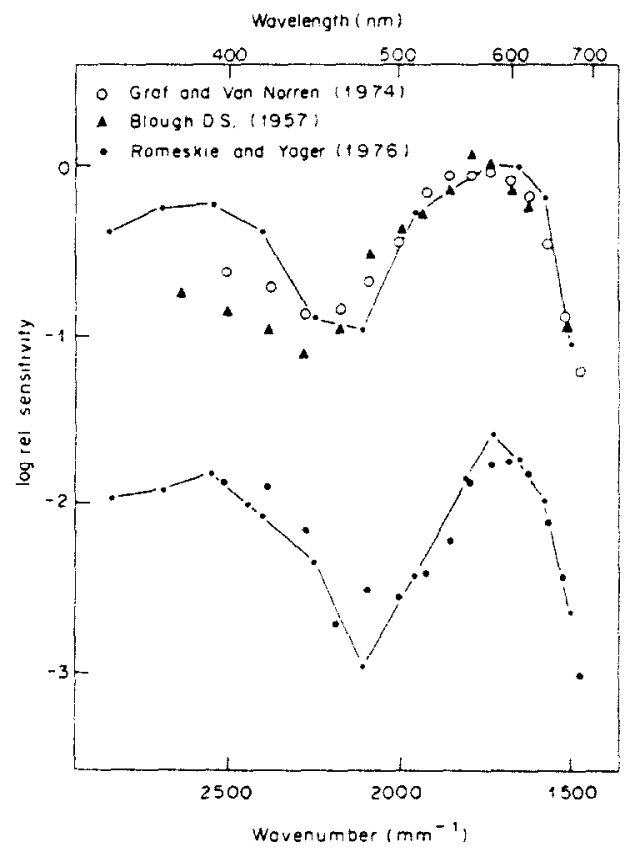

Fig. 8. Results of the criterion response method (lines) compared with spectral sensitivity functions obtained by Blough (1957) (solid triangles), Graf and Van Norren (1974) (open circles) and Romeskie and Yager (1976) (solid circles) The curves have been shifted ajong the ordinate to obtain the best mutual fit, especially beyond $490 \mathrm{~nm}$. 
phenomenon can only account for a minor part of the near-u.v, sensitivity found with the criterion response method, since this sensitivity appeared to be low in flicker photometry.

The role of the coloured oil-droplets in vision is not yet sufficiently clarified. Wallman (1979) has provided evidence that the coloured oil-droplets of birds improve colour vision. In fact the oil-droplets alter the spectral sensitivity functions of the individual cones by steepening their slopes at the short-wavelength side. In addition, they permit an increase in the number of differently sensitive cone classes in spite of a limited number of cone-pigments, since two or more types of oil-droplet can be associated with the same visual pigment. Both factors may very well improve colour discrimination, for in every spectral region good colour discrimination requires a high rate of change of cone responses with the wavelength.

It has been suggested (Walls and Judd, 1933) that the oil-droplets improve visual acuity by reducing the effects of chromatic aberration of the ocular media and the blue haze, resulting from light, scattered by the air. Due to chromatic aberration short-wave and long-wave light from one object point cannot be focussed simultaneously on the retina. Although the oil-droplets limit the spectral bandwidths of the cones, they do not limit the spectral bandwidth of the retina. Consequently chromatic aberration remains a serious problem, unless the violet sensitive cone system does not play a significant role in the perception of contours. In primates the blue sensitive cone system probably does not contribute much to visual acuity (Zrenner and Gouras, 1981), but this may not apply to the pigeon, since this bird is able to perform some form of pattern discrimination in the near-u.v. (Emmerton, 1983).

Finally, since the oil-droplets lie in several layers at different retinal depths, they may play a role in the reduction of the effects of intra-ocular scattered light, especially in the red field, where the inner segments of cones with a red oil-droplet also contain microdroplets (Pedler and Boyle, 1969). Generally stray light has a more oblique angle of incidence on the retina than image-forming light. Consequently blue and green stray light will be largely absorbed by the microdroplets and the yellow, orange and red oildroplets before it can reach the outer segments of the receptors. Red stray light will be less affected.

Acknowledgements - We thank Dr D. van Norren for his critical comments on the manuscript.

\section{REFERENCES}

Bloch S., Martinoya C. and Rivaud S. (1981) Eye move- ments in pigeons: participation in binocular fixation and visual pursuit. J. Physiol., Lond. 320, 20-21.

Blough D. S. (1957) Spectral sensitivity in the pigeon. J. opt. Soc. Am. 47, 827-833.

Bowmaker J. K. (1977) the visual pigments, oil-droplets and spectral sensitivity of the pigeon. Vision Res. 17, 1129-1138.

Campell H. S. and Smith J. L. (1962) The pharmacology of the pigeon pupil. Archs Ophthal. 67, 501-504.

Donovan W. J. (1978) Structure and function of the pigeon visual system. Physiol. Psychol. 6, 403-437.

Emmerton J. (1983) Pattern discrimination in the nearultraviolet by pigeons. Percept. Psychophys. 34, 555-559.

Geri G. A. (1981) Blue cone input to the photopic ERG of the turtle. Vision Res. 21, 893-896.

Goldsmith T. H. and Collins J. S. (1982) Cone oil-droplets of bird retina. Invest. Ophthal. visual Sci., suppl. 322,189

Govardovskii V. I. and Zueva L. V. (1977) Visual pigments of chicken and pigeon. Vision Res. 17, 537-543.

Graf V. and Van Norren D. (1974) A blue sensitive mechanism in the pigeon retina: $\lambda_{\max } 400 \mathrm{~nm}$. Vision Res. 14 , 1203-1209.

Ikeda H. (1965) The spectral sensitivity of the pigeon (Columba livia). Vision Res. 5, 19-36.

Marshall J., Mellario J. and Palmer D. A. (1973) A schematic eye for the pigeon. Vision Res. 13, 2449-2453.

Martin G. R. and Muntz W. R. A. (1979) Retinal oildroplets and vision in the pigeon (Columbia litia). In Neural Mechanisms of Behavior in the Pigeon (Edited by Granda A. M. and Maxwell J. H.). Plenum Press, New York.

Martinoya C., Rey J. and Bloch S. (1981) Limits of the pigeon's binocular feld and direction for best binocular viewing. Vision Res. 21, 1197-1200.

Norren D. Van (1975) Two short wavelength sensitive cone systems in pigeon, chicken and daw. Vision Res. 15, $1164-1166$.

Nuboer J. F. W., Van Nuys W. M. and Wortel J. F. (1983) Cone systems in the rabit retina revealed by ERG-null. detection. J. comp. Physiol. 151, 347-352.

Nygaard R. W. and Frumkes T. E. (1982) Calibration of the retinal illuminance provided by Maxwellian views. Vision Res. 22, 433-434.

Pedler C. and Boyle M. (1969) Multiple oil-droplets in the photoreceptors of the pigeon. Vision Res. 9 , 525-528.

Romeskie M. and Yager D. (1976) Psychophysical studies of pigeon color vision-I. Photopic spectral sensitivity. Vision Res. 16, 501-505.

Strother G. K. (1963) Absorption spectra of retinal oilglobules in turkey, turtle and pigeon Expl Cell Res. 29, 349-355.

Waelchli G. (1883) Zur Topographie der gerfärbten Kugeln der Vogelnetzhaut. Archs Ophthal. 29, 205-223.

Wallman J. (1979) Role of the retinal oil droplets in the color vision of Japanese quail. In Neural Mechanisms of Behavior in the Pigeon (Edited by Granda A. M. and Maxwell J. H.). Plenum Press, New York.

Walls G. L. and Judd H. D. (1933) The intra-ocular filters of vertebrates $B r . J$. Ophthal. 33, 641-675.

Yazulla S. and Granda A. M. (1973) Opponent-color units in the thalamus of the pigeon (Columba Livia). Vision Res. 13, 1555-1563.

Zrenner E. and Gouras P. (1981) Characteristics of the blue sensitive cone mechanism in primate retinal ganglion cells. Vision Res. 21, 1605-1609. 